\title{
A Retrospective Study of Kidney Stone Recurrence in Adults
}

\author{
Jianfeng Zenga, Shanyun Wang ${ }^{\mathrm{b}}$, Liang Zhong ${ }^{\mathrm{a}}$, Zhifeng Huang ${ }^{\mathrm{a}}$, Ye Zeng ${ }^{\mathrm{a}}$, \\ Dongxiang Zheng ${ }^{\text {a }}$, Weiwei Zouc, Haibiao Lai ${ }^{\mathrm{a}, \mathrm{d}}$
}

\begin{abstract}
Background: Nephrolithiasis or kidney stone disease (KSD) is a common disorder worldwide. Despite the availability of a variety of effective management strategies, KSD recurrence remains a problem. In the present study, we investigated the KSD recurrence after the treatment of the first stone episode.
\end{abstract}

Methods: Medical records of all patients who had KDS treated in our department from January 2012 to January 2016 were retrospectively reviewed. A total of 146 patients who had KDS recurrence were identified. The demographic information, biochemical data, treatment methods, report of hydronephrosis severity and stone chemical compositions were collected and analyzed.

Results: We reported that: 1) Sixty four (43.8\%) out of 146 patients with stone recurrence were overweight or obese; 2) Of all 146 patients with stone recurrence, $86(58.9 \%)$ had hyperlipidemia, 77 (52.4\%) had hyperuricemia and 64 (43.8\%) had hyperglycemia; 3$)$ Mini-invasive methods were mostly used for the treatment of the first stone episode; 4) The most chief complaint differed during the first episode and recurrence; 5) The number of patients who had grades 3 and 4 hydronephrosis was significantly lower during recurrence compared with that in the first stone episode; 6) Seventy nine (54.1\%) patients with recurrence had stone of calcium oxalate.

Conclusions: Majority of patients with KSD recurrence have overweight/obesity, hyperlipidemia, hyperuricemia and hyperglycemia, indicating a role of metabolic disorders in stone recurrence. Miniinvasive methods are the mainstay for the management of the first stone episode. The degree of hydronephrosis is significantly reduced

Manuscript submitted December 27, 2018, accepted January 24, 2019

aDepartment of Urology Surgery, Zhongshan Hospital of Traditional Chinese Medicine Affiliated With Guangzhou University of Chinese Medicine, Zhongshan City, China

bDepartment of Obstetrics and Gynecology, Zhongshan Hospital of Traditional Chinese Medicine Affiliated With Guangzhou University of Chinese Medicine, Zhongshan City, China

${ }^{\mathrm{c}}$ Department of Anesthesiology, Zhongshan Torch Development Zone Hospital, Zhongshan City, China

${ }^{\mathrm{d}}$ Corresponding Author: Haibiao Lai, Department of Urology Surgery, Zhongshan Hospital of Traditional Chinese Medicine Affiliated With Guangzhou University of Chinese Medicine, 3 Kangxin Road, Western District, Zhongshan City 528400, Guangdong Province, China.

Email: laihaibiao@hotmail.com

doi: https://doi.org/10.14740/jocmr3753 during stone recurrence, possibly due to increased awareness of the disease and thereafter the change of lifestyle in patients. Over half of recurrent stones are of calcium oxalate.

Keywords: Kidney stone disease; Recurrence; Metabolic disorders; Renal colic; Hydronephrosis; Calcium oxalate

\section{Introduction}

Nephrolithiasis or kidney stone disease (KSD) is a common disorder estimated to affect approximately $10-12 \%$ of men and $5-6 \%$ of women in Western countries $[1,2]$. In a cross-sectional study, Zeng et al investigated 12,570 individuals during 2013 and 2014, and revealed a prevalence rate of 6.4\% in Chinese population [3]. KDS negatively impacts kidney function, which can result in chronic kidney disease and end-stage renal failure; it is also associated with increased risk of myocardial infarction, peripheral arterial disease and hypertension, leading to substantial financial loss resulting from lost work days and health-care costs [4-10]. Due to lifestyle changes such as lack of physical activity and dietary habits, and global warming, the incidence of KSD has been increasing worldwide in the past decades $[1,2,11-18]$. Genetic factors involving in the pathogenesis of kidney stones have also been recognized [1922].

There are a variety of treatment options for KSD including open surgery, percutaneous nephrolithotomy, super-mini percutaneous nephrolithotomy, ureteroscopy, extracorporeal shock wave lithotripsy and conservative intervention/measures [23-28]. Despite satisfactory outcomes after intervention, the recurrence of KSD is dismally high [29-31]. A recent systematic review has shown that the prevalence rate of KSD in the south region of China is markedly higher than that in the north region [11]. In this study, we aimed to explore the recurrence of KSD in patients who were treated in our hospital that is located in South China.

\section{Patients and Methods}

\section{Patients}

This study was approved by the Medical Research Review Committee of Zhongshan Hospital of Traditional Chinese 
Medicine. The requirement for informed consent was waived due to the retrospective nature of current study. Medical records of all patients who had KDS treated in our department from January 2012 to January 2016 were retrospectively reviewed. A total of 146 patients who had KDS recurrence after treatment of first stone episode were identified. The demographic information, biochemical data, treatment methods, severity of hydronephrosis report and stone chemical composition, were collected and analyzed.

\section{Biochemical testing and analysis of stone composition}

Blood cholesterol, uric acid and fasting glucose levels were detected routinely in our diagnostic laboratory. Stone chemical composition was analyzed using an automatic infrared spectrometer (Blue Maude Scientific Instrument Co., Ltd., Tianjin, China).

\section{Assessment of severity of hydronephrosis}

An alternative grading system was used to assess the severity of hydronephrosis [32]. Grade 0 (normal) suggests no hydronephrosis; grade 1 is defined as having dilatation of renal pelvis alone; grade 2 has dilatation of renal pelvis plus caliceal dilatation; grades 1 and 2 indicate mild hydronephrosis; grade 3 means moderate hydronephrosis where $<1 / 2$ renal parenchymal loss is seen; and grade 4 is severe hydronephrosis, which has $>1 / 2$ renal parenchymal loss.

\section{Statistical analysis}

Data normality was assessed by the Shapiro-Wilk test. Normally distributed continuous variables were presented as mean \pm standard deviation (SD) and tested by Student's $t$-test. Categorical variables were presented by numbers (percentages) and tested by Chi-square test. $\mathrm{P}<0.05$ was considered statistically significant.

\section{Results}

A total of 3,985 patients had KSD treated in our department from January 2012 to January 2016. Of these patients, 146 (3.7\%) including 100 males and 46 females had stone recurrence. The mean age of male patients was $39.4 \pm 15.8$ years, which was not significantly different than $41.5 \pm 18.5$ years of female patients. The distribution of the body mass index (BMI) of these 146 patients is shown in Figure 1. According to the criteria set for Chinese population, i.e., a BMI $\geq 24 \mathrm{~kg} / \mathrm{m}^{2}$ is regarded as overweight or obesity [33], 64 (43.8\%) patients are overweight or obese. Among these 146 patients, 86 had hyperlipidemia, 77 had hyperuricemia and 64 had hyperglycemia. The most-used procedure for the management of the first stone episode was percutaneous nephrolithotomy (65 cases) followed by conservative treatment (34 cases), extracorporeal

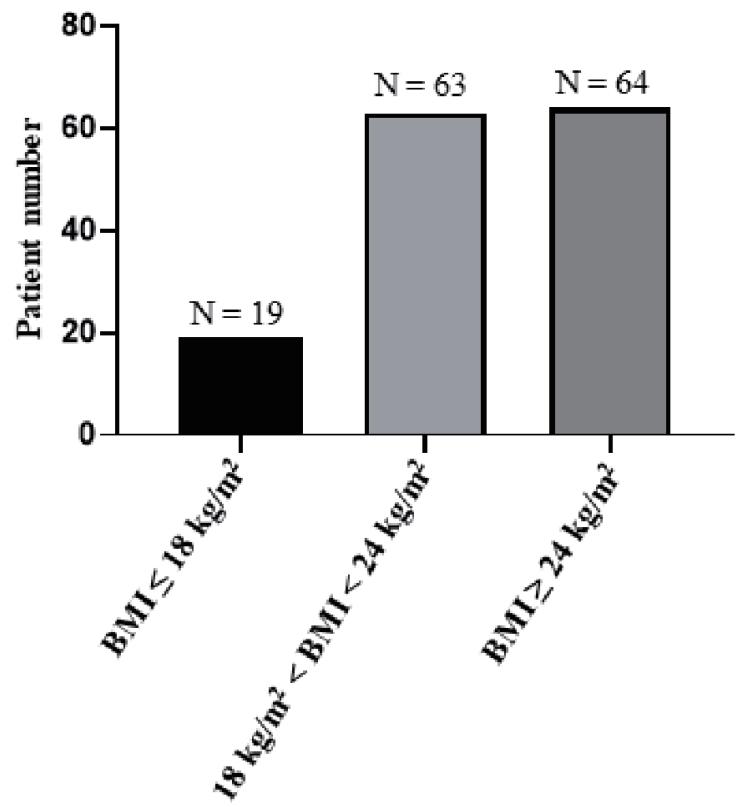

Figure 1. Distribution of body mass index of patients with recurrent KDS. As shown in this figure, 64 patients had an abnormally high body mass index (BMI).

shock wave lithotripsy (22 cases), super-mini percutaneous nephrolithotomy (20 cases), open surgery (four cases) and ureteroscopy (one case).

During the first stone episode the most chief complaint was renal colic (Fig. 2a), and in contrast, the most chief complaint for patients with recurrence was the symptom associated with infection (Fig. 2b). We compared the severity of hydronephrosis during the first stone episode and recurrence in those 146 patients, and found that the number of patients who had grades 3 and 4 hydronephrosis was significantly lower during recurrence compared with that during the first stone episode (Table 1). Chemical composition analysis showed that most recurrent stones were of calcium oxalate followed by calcium oxalate plus hydroxyapatite, calcium oxalate plus magnesium ammonium phosphate and uric acid plus calcium oxalate (Table 2).

\section{Discussion}

In this study, we retrospectively reviewed medical records and identified 146 cases of stone recurrence in a total of 3,985 patients from January 2012 to January 2016. We reported that: 1) Sixty four $(43.8 \%)$ out of 146 patients with stone recurrence were overweight or obese; 2) Of all 146 patients with stone recurrence, 86 (58.9\%) had hyperlipidemia, $77(52.4 \%)$ had hyperuricemia and 64 (43.8\%) had hyperglycemia; 3) Miniinvasive methods were mostly used for the treatment of the first stone episode; 4) The most chief complaint differed during the first episode and recurrence; 5) The number of patients who had grades 3 and 4 hydronephrosis was significantly lower during recurrence compared with that during the first stone 
a

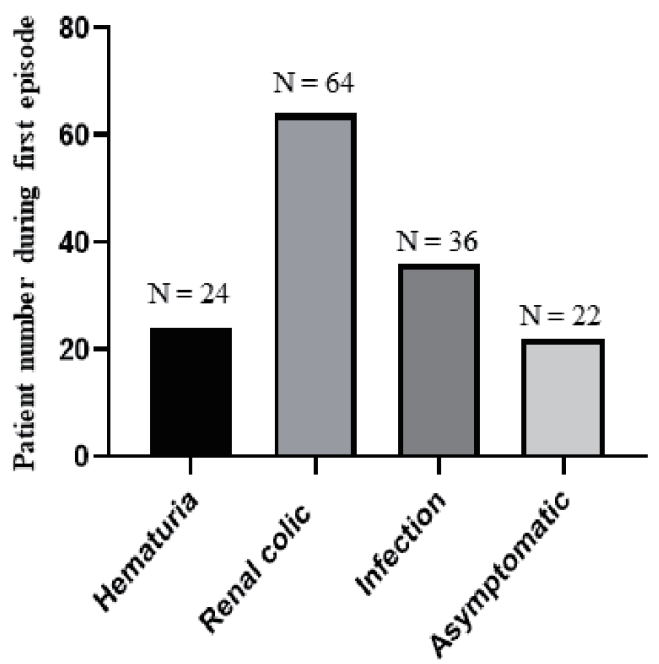

b

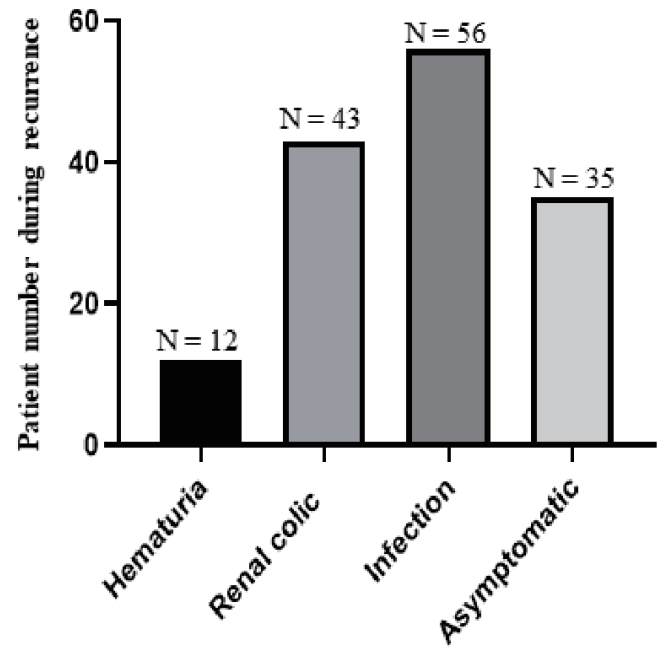

Figure 2. Chief complaint during the first stone episode and recurrence. As shown in this figure, the most chief complaint was renal colic for patients with first stone episode (a). In contrast, the most chief complaint for patients during recurrence was the symptom associated with infection (b).

episode; 6) Seventy nine (54.1\%) patients with recurrence had stone of calcium oxalate.

Varied recurrence rates of KSD have been reported from study to study but are dismally high. In a retrospective study, Rule et al observed that KSD recurrence rates at 2, 5, 10, and 15 years were $11 \%, 20 \%, 31 \%$, and $39 \%$, respectively [30]. By following up of 300 consecutive patients for 7 to 17 years, Trinchieri et al reported a $27 \%$ symptomatic stone recurrence [34]. Vaughan et al looked at a random sample of incident symptomatic kidney stone formers consisting of 3,364 patients, and found that the stone recurrence rates per 100 person-years were 3.4 after the first episode, 7.1 after the second episode, 12.1 after the third episode, and 17.6 after the fourth or higher episode [35]. Our observational period ranges from 2 - 6 years, but the data on the number of episodes for each indi- vidual are not available, making it difficult to compare our results with Vaughan's. Nevertheless, a recurrence rate of $3.7 \%$ observed by us is low, which probably is due to the education we provided that increased patients' awareness of dietary role in the prevention of KSD recurrence and thereafter the change of lifestyle.

In 146 patients who had KSD recurrence, 100 (68.5\%) are males, which is in line with the finding that KSD occurs more frequently in men than in women [11,36, 37]. Obesity has been found associated positively with KSD. Taylor et al revealed that the risk factor for KSD for men with a BMI of 30 or greater versus those with a BMI of 21 to 22.9 was 1.33 , and the risk factor for the same categories of BMI in older and younger women was 1.90 [16]. Increased BMI as a risk factor for kidney stones has also been demonstrated by

Table 1. Number of Patients Who Had Different Grades of Hydronephrosis in the First Stone Episode and Recurrence

\begin{tabular}{lllll} 
& Normal (Grade 0) & Mild (Grades 1 and 2) & Moderate (Grade 3) & Severe (Grade 4) \\
\hline First episode (n) & $17(11.64 \%)$ & $62(42.47 \%)$ & $34(23.29 \%)$ & $33(22.60 \%)$ \\
Recurrence (n) & $26(17.81 \%)$ & $87(59.59 \%)$ & $16(10.96 \%)^{*}$ & $17(11.64 \%)^{*}$ \\
\hline
\end{tabular}

${ }^{*} \mathrm{P}<0.05$ compared with the number of patients who had the corresponding grade in the first stone episode.

Table 2. Stone Composition in Recurrent KSD

\begin{tabular}{ll}
\hline Stone composition & Number of patients \\
\hline Calcium oxalate & $79^{*}$ \\
Calcium oxalate + hydroxyapatite & 21 \\
Calcium oxalate + magnesium ammonium phosphate & 17 \\
Uric acid & 8 \\
Uric acid + calcium oxalate & 15 \\
Uric acid + calcium oxalate + hydroxyapatite & 6 \\
\hline
\end{tabular}

${ }^{*} \mathrm{P}<0.05$ compared with the number of patients in any of other groups. 
others $[38,39]$. In our series, $43.8 \%$ patients had abnormally high BMI, supporting the notion that BMI is a risk factor for KSD. The links between metabolic syndrome and KSD has been established [40]. Diabetic patients have a higher risk of suffering from KSD [14]. In our series, $43.8 \%$ of patients had hyperglycemia. Additionally, half patients had hyperuricemia and approximately $60 \%$ of patients had hyperlipidemia. These findings suggest that control of metabolic disorders might be pivotal for the prevention of KSD recurrence.

In our practice, we recommend patients change diet, do more exercise, lose weight and take more fluids, which might contribute to the low recurrence rate observed in this study. This is a retrospective study and the inherent bias might overestimate the results. Additionally, the sample size is small, which might limit the generalizability of our findings. These are the limitations of this study.

\section{Conclusions}

In conclusion, the majority of patients with KSD recurrence have overweight/obesity, hyperlipidemia, hyperuricemia and hyperglycemia, indicating a role of metabolic disorders in stone recurrence. These data suggest that life style modification and dietary intervention are important in the prevention of KSD recurrence. Mini-invasive methods are the mainstay for the management of the first stone episode. The degree of hydronephrosis is significantly reduced during stone recurrence, possibly due to increased awareness of the disease and thereafter the change of lifestyle in patients. Over half of recurrent stones are of calcium oxalate.

\section{Funding Disclosure}

There is no funding received for this study.

\section{Conflict of Interest}

None.

\section{Reference}

1. Stamatelou KK, Francis ME, Jones CA, Nyberg LM, Curhan GC. Time trends in reported prevalence of kidney stones in the United States: 1976-1994. Kidney Int. 2003;63(5):1817-1823.

2. Edvardsson VO, Indridason OS, Haraldsson G, Kjartansson O, Palsson R. Temporal trends in the incidence of kidney stone disease. Kidney Int. 2013;83(1):146-152.

3. Zeng G, Mai Z, Xia S, Wang Z, Zhang K, Wang L, Long $Y$, et al. Prevalence of kidney stones in China: an ultrasonography based cross-sectional study. BJU Int. 2017;120(1):109-116.

4. Sigurjonsdottir VK, Runolfsdottir HL, Indridason OS, Palsson R, Edvardsson VO. Impact of nephrolithiasis on kidney function. BMC Nephrol. 2015;16:149.

5. El-Zoghby ZM, Lieske JC, Foley RN, Bergstralh EJ, Li X, Melton LJ, 3rd, Krambeck AE, et al. Urolithiasis and the risk of ESRD. Clin J Am Soc Nephrol. 2012;7(9):14091415.

6. Worcester EM, Parks JH, Evan AP, Coe FL. Renal function in patients with nephrolithiasis. J Urol. 2006;176(2):600603; discussion 603.

7. Rule AD, Roger VL, Melton LJ, 3rd, Bergstralh EJ, Li X, Peyser PA, Krambeck AE, et al. Kidney stones associate with increased risk for myocardial infarction. J Am Soc Nephrol. 2010;21(10):1641-1644.

8. Cupisti A, Morelli E, Meola M, Cozza V, Parrucci M, Barsotti G. Hypertension in kidney stone patients. Nephron. 1996;73(4):569-572.

9. Kittanamongkolchai W, Mara KC, Mehta RA, Vaughan LE, Denic A, Knoedler JJ, Enders FT, et al. Risk of Hypertension among First-Time Symptomatic Kidney Stone Formers. Clin J Am Soc Nephrol. 2017;12(3):476-482.

10. Fan X, Kalim S, Ye W, Zhao S, Ma J, Nigwekar SU, Chan $\mathrm{KE}$, et al. Urinary stone disease and cardiovascular disease risk in a rural Chinese population. Kidney Int Rep. 2017;2(6):1042-1049.

11. Wang W, Fan J, Huang G, Li J, Zhu X, Tian Y, Su L. Prevalence of kidney stones in mainland China: A systematic review. Sci Rep. 2017;7:41630.

12. Romero V, Akpinar H, Assimos DG. Kidney stones: a global picture of prevalence, incidence, and associated risk factors. Rev Urol. 2010;12(2-3):e86-96.

13. Robertson WG, Heyburn PJ, Peacock M, Hanes FA, Swaminathan $\mathrm{R}$. The effect of high animal protein intake on the risk of calcium stone-formation in the urinary tract. Clin Sci (Lond). 1979;57(3):285-288.

14. Aune D, Mahamat-Saleh Y, Norat T, Riboli E. Body fatness, diabetes, physical activity and risk of kidney stones: a systematic review and meta-analysis of cohort studies. Eur J Epidemiol. 2018;33(11):1033-1047.

15. Carbone A, Al Salhi Y, Tasca A, Palleschi G, Fuschi A, De Nunzio C, Bozzini G, et al. Obesity and kidney stone disease: a systematic review. Minerva Urol Nefrol. 2018;70(4):393-400.

16. Taylor EN, Stampfer MJ, Curhan GC. Obesity, weight gain, and the risk of kidney stones. JAMA. 2005;293(4):455462.

17. Fakheri RJ, Goldfarb DS. Ambient temperature as a contributor to kidney stone formation: implications of global warming. Kidney Int. 2011;79(11):1178-1185.

18. Straub M, Hautmann RE. Developments in stone prevention. Curr Opin Urol. 2005;15(2):119-126.

19. Konrad M, Hou J, Weber S, Dotsch J, Kari JA, Seeman T, Kuwertz-Broking E, et al. CLDN16 genotype predicts renal decline in familial hypomagnesemia with hypercalciuria and nephrocalcinosis. J Am Soc Nephrol. 2008;19:171-181.

20. Beara-Lasic L, Edvardsson V, Palsson R, Lieske J, Goldfarb D, Milliner D. Genetic causes of kidney stones and kidney failure. Clin Rev Bone Miner Metab. 2011;10(6):118.

21. Li X, Dang X, Cheng Y, Zhang D, Zhang X, Zou T, Xing 
J. Common variants in ALPL gene contribute to the risk of kidney stones in the Han Chinese population. Genet Test Mol Biomarkers. 2018;22(3):187-192.

22. Edvardsson VO, Goldfarb DS, Lieske JC, Beara-Lasic L, Anglani F, Milliner DS, Palsson R. Hereditary causes of kidney stones and chronic kidney disease. Pediatr Nephrol. 2013;28(10):1923-1942.

23. Zhang Y, Wei C, Pokhrel G, Liu X, Ouyang W, Gan J, Ding B, et al. Mini-percutaneous nephrolithotomy with ureter catheter: a safe and effective form of mPCNL offers better quality of life. Urol Int. 2018:1-7.

24. York NE, Zheng M, Elmansy HM, Rivera ME, Krambeck AE, Lingeman JE. Stone-free outcomes of flexible ureteroscopy for renal calculi utilizing computed tomography imaging. Urology. 2018.

25. Zeng G, Wan S, Zhao Z, Zhu J, Tuerxun A, Song C, Zhong L, et al. Super-mini percutaneous nephrolithotomy (SMP): a new concept in technique and instrumentation. BJU Int. 2016;117(4):655-661.

26. Simayi A, Liu Y, Yiming M, AlSmadi J, Yusufu A, Alimu Y, Lei P, et al. Clinical application of super-mini PCNL (SMP) in the treatment of upper urinary tract stones under ultrasound guidance. World J Urol. 2018.

27. Reesink DJ, Scheltema JMW, Barendrecht MM, Boeken Kruger AE, Jansonius A, Wiltink J, van der Windt F. Extracorporeal shock wave lithotripsy under intravenous sedation for treatment of urolithiasis. Scand J Urol. 2018:16.

28. Sorokin I, Pearle MS. Medical therapy for nephrolithiasis: State of the art. Asian J Urol. 2018;5(4):243-255.

29. Zisman AL. Effectiveness of treatment modalities on kidney stone recurrence. Clin J Am Soc Nephrol. 2017;12(10):1699-1708.

30. Rule AD, Lieske JC, Li X, Melton LJ, 3rd, Krambeck AE, Bergstralh EJ. The ROKS nomogram for predicting a second symptomatic stone episode. J Am Soc Nephrol. 2014;25(12):2878-2886.
31. Ettinger B, Citron JT, Livermore B, Dolman LI. Chlorthalidone reduces calcium oxalate calculous recurrence but magnesium hydroxide does not. J Urol. 1988;139(4):679684.

32. Onen A. An alternative grading system to refine the criteria for severity of hydronephrosis and optimal treatment guidelines in neonates with primary UPJ-type hydronephrosis. J Pediatr Urol. 2007;3(3):200-205.

33. Lin WY, Tsai SL, Albu JB, Lin CC, Li TC, Pi-Sunyer FX, Sung PK, et al. Body mass index and all-cause mortality in a large Chinese cohort. CMAJ. 2011;183(6):E329-336.

34. Trinchieri A, Ostini F, Nespoli R, Rovera F, Montanari E, Zanetti G. A prospective study of recurrence rate and risk factors for recurrence after a first renal stone. J Urol. 1999;162(1):27-30.

35. Vaughan LE, Enders FT, Lieske JC, Pais VM, Rivera ME, Mehta RA, Vrtiska TJ, et al. Predictors of symptomatic kidney stone recurrence after the first and subsequent episodes. Mayo Clin Proc. 2019;94(2):202-210.

36. Dall'era JE, Kim F, Chandhoke PS. Gender Differences among Hispanics and Caucasians in symptomatic presentation of kidney and ureteral stones. J Endourol. 2005;19(3):283-286.

37. Pfau A, Knauf F. Update on nephrolithiasis: core curriculum 2016. Am J Kidney Dis. 2016;68(6):973-985.

38. Yoshimura E, Sawada SS, Lee IM, Gando Y, Kamada M, Matsushita M, Kawakami R, et al. Body mass index and kidney stones: a cohort study of Japanese men. J Epidemiol. 2016;26(3):131-136.

39. Sorensen MD, Chi T, Shara NM, Wang H, Hsi RS, Orchard T, Kahn AJ, et al. Activity, energy intake, obesity, and the risk of incident kidney stones in postmenopausal women: a report from the Women's Health Initiative. J Am Soc Nephrol. 2014;25(2):362-369.

40. Goldfarb DS, Arowojolu O. Metabolic evaluation of firsttime and recurrent stone formers. Urol Clin North Am. 2013;40(1):13-20. 